\title{
Contact Transformation for the Prediction of Structural Wears
}

\author{
James Shih-Shyn $\mathrm{Wu}^{1, \mathrm{a}}$, Yi-Tsung Lin ${ }^{1, \mathrm{~b}}$ and Yuan-Lung Lai ${ }^{2, \mathrm{c}^{*}}$ \\ ${ }^{1}$ Institute of Mechanical Engineering, National Chung-Hsing University, Taichung, Taiwan, R.O.C. \\ ${ }^{2}$ Department of Industrial Education and Technology, National Changhua University of Education, \\ Changhua, Taiwan, R.O.C. \\ asswu@dragon.nchu.edu.tw, bytlin721@gmail.com, 'lyllaiber@cc.ncue.edu.tw
}

Keywords: Finite element, Sliding wear, Wear simulation, Contact transformation.

\begin{abstract}
In prediction of structural wears with the finite element (FE) approach, the geometry of FE model must be updated constantly to imitate the nonlinear wear behaviors which need a great amount of computational time. Improvement of efficiency of wear simulations becomes increasingly important due to the design requirements. In the current study, an alternative and efficient FE approach by introducing the approach of contact surface transformation is proposed to improve the simulation efficiency of structural wear. The FE model of pin-on-plate wear problem is demonstrated and wear result presented is comparable to available simulation data. The result shows that the computational time is comparatively reduced and numerical precision is increased. It is believed that the current method can offer superior performance for larger and complex structures.
\end{abstract}

\section{Introduction}

Wear is the most significant factor in reducing the service life of mechanical components. In order to improve the service life of components, understanding of the wear behavior of mechanical components is a significant task for engineering design. The FE model has been developed using Archard's wear law [1] in order to simulate large or complex structures and provide more valuable information of structural wear for engineering design [2-5]. In the FE model of wear prediction, the geometry of contact regions between components is variable and the wear behavior is generally nonlinear. The geometry at the end of each sliding motion needs to be updated dynamically [3] and much more computational time in the iterative procedures is required.

Several ideas have been offered in an attempt to reduce computational time of the wear simulation. Põdra and Andersson [2] proposed an incremental formulation with an optimal time step (such as scaling approach) to obtain more computational efficiency for the pin-on-plate wear simulation. Sfantos and Aliabadi [6] indicated that BEM can reduce the number of unknowns in wear simulation, making updating of the worn components relatively fast and easy. However, BEM uses an asymmetric matrix, which is more complicated than approaches using a symmetric matrix. Recently, Mukras et al. [7] proposed parallel computation using nonlinear FE analysis in order to minimize the computational time with the accuracy and stability of wear prediction. However, the computational time of matrix operations were still longer, especially in the large structures. Bae et al. [8] used a substructure method to develop an FEM based approach to wear simulation that can reduce a large number of the unknowns required by traditional FEM, significantly reducing computational time, but the matrix of overall structures needs to change repeatedly in the iterative procedures. Therefore, reducing the computation time of wear simulation remains worthy of study.

Wear formulation. The wear depth is of more interest from the viewpoint of engineering, and can be expressed as:

$$
d h^{p}=\frac{d V^{p}}{A_{c}}=\frac{k_{w}^{p} F_{n}^{p}\left(x_{j}\right) d S^{p}}{A_{c}} .
$$


where superscript $p=\{a, b\}$ is the component index, $j$ is the index of the contact point, $h$ the wear depth, $S$ the sliding distance, $k_{w}$ is the wear coefficient in units of $\mathrm{mm}^{3} / \mathrm{N}-\mathrm{m}$, which can be determined via experiment, $F_{n}\left(x_{j}\right)$ is the normal force on contact point $x_{j}$, and $A_{c}$ is the contact area.

Contact formulation. During the wear motion, the geometry and the normal forces on the contact region change, but the contact force throughout the contact region must always be at equilibrium. A modified localized Lagrange multiplier method (MLLM) is defined. It consists of a governing equation for the contact problem and constrains conditions on the contact region as follows:

$$
\begin{aligned}
\mathbf{K}^{a} \mathbf{u}^{a}+\mathbf{B}^{a} \lambda_{c}^{a}=\mathbf{F}_{e x t}^{a} . \\
\mathbf{K}^{b} \mathbf{u}^{b}+\mathbf{B}^{b} \lambda_{c}^{b}=\mathbf{F}_{\text {ext }}^{b}
\end{aligned}
$$

and

$$
\begin{aligned}
& \mathbf{B}^{a T} \mathbf{u}^{a}-\mathbf{u}_{c}=\mathbf{g} \\
& \mathbf{B}^{b T} \mathbf{u}^{b}-\mathbf{u}_{c}=-\mathbf{g} \cdot \\
& \lambda_{c}^{a}+\lambda_{c}^{b}=0
\end{aligned}
$$

where superscript $T$ stands for the transpose of the specified matrix, $\mathbf{B}$ is a Boolean matrix that extracts the degrees of freedom (DOF) on the contact region of components $a$ and $b . \lambda_{c}$ is the Lagrange multipliers [9] that correspond to local contact forces acting on the contact region. $\mathbf{u}_{c}$ is the local displacement on the contact region and $\mathbf{g}$ is the initial gap vector between components on the contact region. Here, the components are assumed to have tight contact without gaps, i.e., $\mathbf{g}=\mathbf{0}$.

After manipulations, the displacement of component $b$ may be expressed based on Eq. (2) and plugging the displacement equation into the second equation in Eq. (3) yields

$$
\mathbf{B}^{b T}\left(\mathbf{K}^{b}\right)^{-1} \mathbf{B}^{b} \boldsymbol{\lambda}_{c}^{b}+\mathbf{u}_{c}=\mathbf{B}^{b T}\left(\mathbf{K}^{b}\right)^{-1} \mathbf{F}_{\text {ext }}^{b} .
$$

According to the operation of the Boolean matrix, matrix operation can be used to consider the local contact DOF only. Thus, the partitioned inversion algorithm may be applied, and the matrix and force vector are partitioned into non-contact sub-matrix $\mathbf{k}_{b b}^{b}$, contact sub-matrix $\mathbf{k}_{c c}^{b}$, sub-vector $\mathbf{f}_{b b}^{b}$ corresponds to load on the external non-contact DOF, while the contact DOF on external feature is ignored, that is

$$
\left(\mathbf{K}^{b}\right)^{-1}=\left[\begin{array}{ll}
\mathbf{k}_{b b}^{b} & \mathbf{k}_{b c}^{b} \\
\mathbf{k}_{c b}^{b} & \mathbf{k}_{c c}^{b}
\end{array}\right]^{-1}=\left[\begin{array}{ll}
\hat{\mathbf{k}}_{b b}^{b} & \hat{\mathbf{k}}_{b c}^{b} \\
\hat{\mathbf{k}}_{c b}^{b} & \hat{\mathbf{k}}_{c c}^{b}
\end{array}\right], \mathbf{F}_{\text {ext }}^{b}=\left[\begin{array}{c}
\mathbf{f}_{b b}^{b} \\
\mathbf{0}
\end{array}\right] .
$$

Insertion of Eq. (5) into Eq. (4), $\mathbf{u}_{c}=\mathbf{Q}^{a T} \mathbf{u}^{a}$ and $\lambda_{c}^{b}=-\lambda_{c}^{a}$ can be obtained from Eq. (3). Thus the local contact forces $\left(\boldsymbol{\lambda}_{c}\right)$ may be derived as

$$
\begin{aligned}
& \lambda_{c}^{a}=-\left(\hat{\mathbf{k}}_{c c}^{b}\right)^{-1} \hat{\mathbf{k}}_{c b}^{b} \mathbf{f}_{b b}^{b}+\left(\hat{\mathbf{k}}_{c c}^{b}\right)^{-1} \mathbf{B}^{a T} \mathbf{u}^{a} . \\
& \lambda_{c}^{b}=\left(\hat{\mathbf{k}}_{c c}^{b}\right)^{-1} \hat{\mathbf{k}}_{c b}^{b} \mathbf{f}_{b b}^{b}-\left(\hat{\mathbf{k}}_{c c}^{b}\right)^{-1} \mathbf{B}^{a T} \mathbf{u}^{a}
\end{aligned} .
$$

Combining Eqs. (2) and (6) produces a set of new coupled equations, which are used to solve the contact behavior in the wear simulation

$$
\begin{aligned}
\overline{\mathbf{K}}^{a} \mathbf{u}^{a} & =\overline{\mathbf{F}}_{\text {ext }}^{a} . \\
\mathbf{K}^{b} \mathbf{u}^{b} & =\overline{\mathbf{F}}_{\text {ext }}^{b} .
\end{aligned}
$$

where 


$$
\begin{aligned}
\overline{\mathbf{K}}^{a} & =\mathbf{K}^{a}+\mathbf{B}^{a}\left(\hat{\mathbf{k}}_{c c}^{b}\right)^{-1} \mathbf{B}^{a T} \\
\overline{\mathbf{F}}_{\text {ext }}^{a} & =\mathbf{F}_{\text {ext }}^{a}+\mathbf{B}^{a}\left(\left(\hat{\mathbf{k}}_{c c}^{b}\right)^{-1} \hat{\mathbf{k}}_{c b}^{b} \mathbf{f}_{b b}^{b}\right) \\
\overline{\mathbf{F}}_{\text {ext }}^{b} & =\mathbf{F}_{\text {ext }}^{b}-\mathbf{B}^{b}\left(\left(\hat{\mathbf{k}}_{c c}^{b}\right)^{-1} \hat{\mathbf{k}}_{c b}^{b} \mathbf{f}_{b b}^{b}-\left(\hat{\mathbf{k}}_{c c}^{b}\right)^{-1} \mathbf{B}^{a T} \mathbf{u}^{a}\right)
\end{aligned} .
$$

The contact normal force can be derived by introducing a normal vector $\mathbf{n}$ of contact node in component $p=\{a, b\}$ as $F_{n}^{p}\left(x_{j}\right)=\lambda_{c}^{p T}\left(x_{j}\right) \mathbf{n}^{p}\left(x_{j}\right)$. Finally, an implicit formula is used to integrate Eq. (1); the wear depth can be written as

$$
h_{i+1}^{p}\left(x_{j}\right)=h_{i}^{p}\left(x_{j}\right)+\frac{k_{w}^{p} \lambda_{c}^{p T}\left(x_{j}\right) \mathbf{n}^{p}\left(x_{j}\right) S_{i+1}^{p}}{A_{c, i+1}} .
$$

where subscript $i$ is the incremental step index and $j$ is the contact node index. The second term on the right side represent the excessive wear results generated at incremental step $i+1$ which can be used to update the geometry of the worn components. The contact area $\left(A_{c}\right)$ is calculated using the numerical integration of Gaussian quadrature.

In the current study, an iterative process was used that considers nonlinear wear and the motion of cyclic slide with an incremental change. In each step, geometry of the worn component needs to be updated and elements on the contact region are varied subsequently. The determinant weighted algorithm is adopted to automatically re-mesh the FE model of the worn component in the current study. Once the normal forces on the contact region are evaluated, the wear depth may be finally estimated using the current method.

Result and discussion. To test the current method, the pin-on-plate wear problem is used. The wear results and the computational time of the current method are compared with those of the previous method [3]. Here, only wear on the pin is considered, and the pin moves on the plate along a circular route with a radius of $5 \mathrm{~mm}$. The geometry of the model is shown in Fig. 1.

In this study, the hexahedron linear element is used with three translational displacements at each node to reconstruct the FE models. The ultra-high molecular weight polyethylene (UHMWPE) pin is designated $a$, and discretized into 1331 nodes and 1080 elements. The load applied to the top of the pin is $70.7 \mathrm{~N}$. The 316L ASTM F318 annealed stainless steel plate is designated $b$, and discretized into 530 nodes and 252 elements. The motion of the bottom of the plate is restrained, as shown in Fig. 1. The corresponding material properties, Young' modulus $(E)$ and Poisson's ratio $(v)$, are $0.8 \mathrm{GPa}$ and 0.47 for the UHMWPE and $190 \mathrm{GPa}$ and 0.305 for the stainless steel, respectively. For the contact conditions, complete contact is assumed between the pin and plate, as the initial condition. For the wear parameters, the wear coefficient is $0.8 \times 10^{-6} \mathrm{~mm}^{3} / \mathrm{N}-\mathrm{m}$ and the total sliding cycle is 1 million cycles [3].
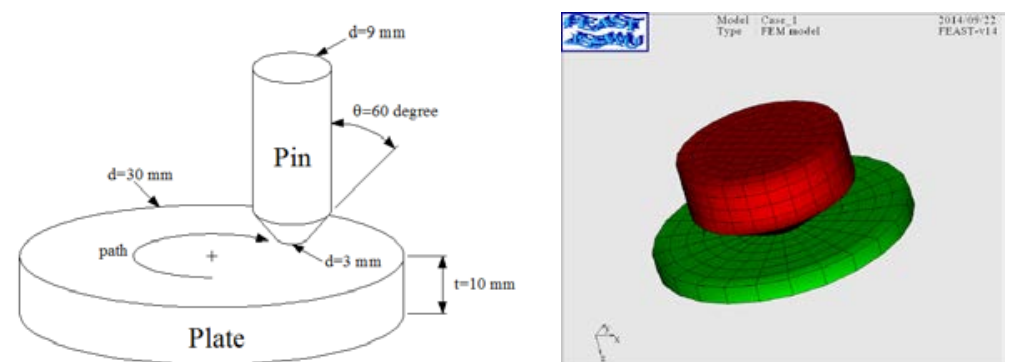

Fig. 1. A wear behavior of pin-on-plate as defined by Wu et al. [3] and FE model.

When the number of increments is 10, Fig. 2 shows the curves of the wear depth for the previous and current method. The wear depth is $0.1395 \mathrm{~mm}$ for previous method and $0.1401 \mathrm{~mm}$ for the current method. When the number of increments is 100 , the wear depth of $0.1374 \mathrm{~mm}$ in the current method is in good agreement with the previous method [3]. In order to demonstrate the characteristics of the current method, previous method is used in state 1, while current method is used in state 2 . Since the whole matrix in state 1 needs to be regenerated and decomposed after updating the geometry, state 1 is taken as the benchmark and the run time ratio is calculated. Fig. 3 shows the 
comparison of the run time ratio in the previous and current method, and the run time ratio gradually decreases and converges to approximately 0.53 . The finding tells us that the simulation efficiency obtained from the current is great improved than the traditional FE approaches, and the round-of errors of data in matrix are decreased through the reduction of flops (floating-point operations).

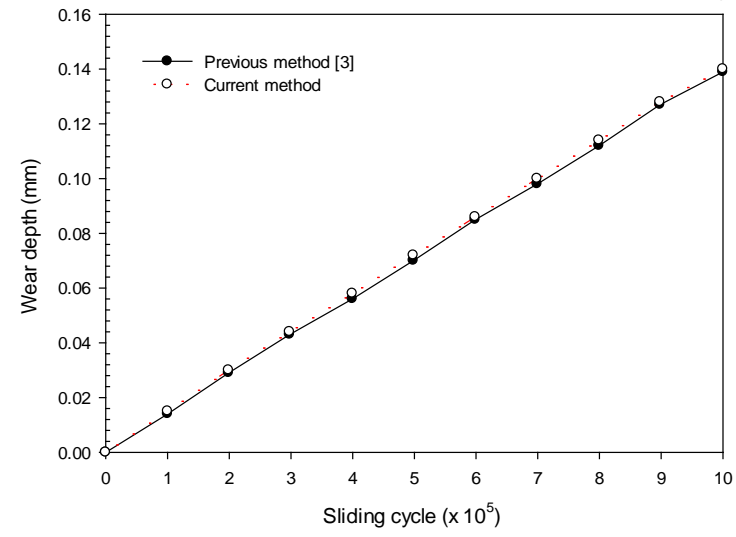

Fig. 2. Wear depth of pin

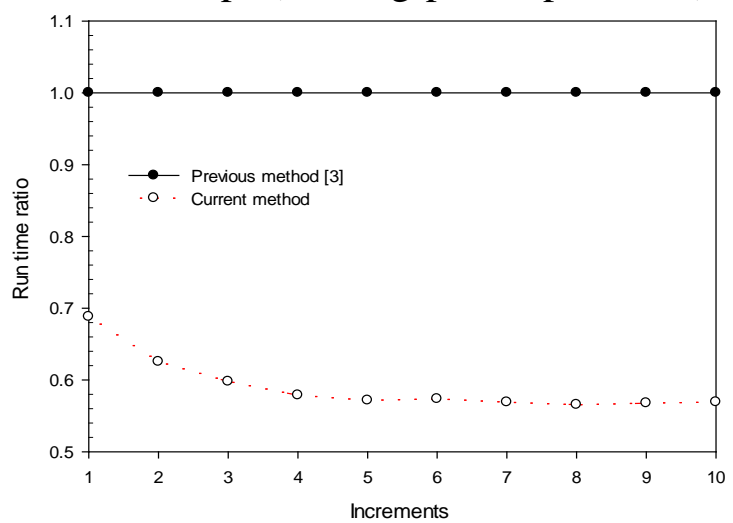

Fig. 3. Run time ratio

\section{Conclusions}

The current study reveals that the proposed method can improve the simulation efficiency of structural wear. In the iterative procedures, the operation of the inverse of a large matrix for the classic methods may have a significant computational error and computational time. However, the contact transformation in the current study is used to avoid the calculation of whole structural matrix and can be used to consider the local contact DOF of matrices. For the wear simulation, this is a great advantage over the classic FE method, especially when the FE model is updated after every step. To demonstrate the validation of the current method, FE models of pin-on-plate is demonstrated. The results indicate that the computational time can be reduced significantly without loss of accuracy and is in significant agreement with the published literatures. It is believed that the current method can offer superior performance for larger and complex structures.

\section{Acknowledgements}

The authors gratefully acknowledge the supports of the National Science Council in Taiwan through project number NSC 99-2221-E-005-048-MY310 and the Mechanical and System Research Laboratories, Industrial Technology Research Institute in Taiwan.

\section{References}

[1] J.F. Archard: J. Appl. Phys. Vol. 24 (1953), p. 981.

[2] P. Põdra and S. Andersson:Tribol. Int. Vol. 32 (1999), p. 71.

[3] J.S.-S. Wu, J.-P. Hung, C.-S. Shu and J.-H. Chen: Comput. Meth. Programs Biomed. Vol. 70 (2003), p. 81.

[4] A.R. AbuBakar and H. Ouyang: Wear Vol. 264 (2008), p. 1069.

[5] R.D. Queiroz, A.L.L. Oliveira, F.C. Trigo and J.A. Lopes: Wear Vol. 298-299 (2013), p. 8.

[6] G.K. Sfantos and M.H. Aliabadi: Wear Vol. 260 (2006), p. 1119.

[7] S. Mukras, N.H. Kim, W.G. Sawyer, D.B. Jackson and L.W. Bergquist: Wear Vol. 266(7-8) (2009), p. 822.

[8] J.W. Bae, C.Y. Lee and Y.S. Chai: Int. J. Precis. Eng. Manuf. Vol. 10(4) (2009), p. 63.

[9] C. Oysu: Appl. Math. Modell. Vol. 31 (2007), p. 2744. 